\title{
How far should we go in optimal cytoreductive surgery for ovarian cancer?
}

\author{
Luis A. Hernandez-Lopez ${ }^{1,2}$, Angel Elizalde-Mendez ${ }^{1}$
}

${ }^{1}$ Gynaecological Oncology Department, Instituto Nacional de Perinatologia, Mexico City, Mexico; ${ }^{2}$ Surgical Gynaecology, Instituto Nacional de Perinatologia, Mexico City, Mexico

Contributions: (I) Conception and design: LA Hernandez-Lopez; (II) Administrative support: A Elizalde-Mendez; (III) Provision of study materials or patients: A Elizalde-Mendez; (IV) Collection and assembly of data: A Elizalde-Mendez; (V) Data analysis and interpretation: LA Hernandez-Lopez; (VI) Manuscript writing: All authors; (VII) Final approval of manuscript: All authors.

Correspondence to: Luis A. Hernandez-Lopez. Montes Urales 800, Lomas Virreyes, Miguel Hidalgo, 11000, Mexico City, Mexico. Email: luis.hernandezlopez@inper.gob.mx.

\begin{abstract}
The treatment of ovarian cancer should be appropriate, since clinical and surgical decisions may affect the prognosis; the surgery must be performed by an expert oncological surgeon or gynecological oncologist, it's fundamental roles are cancer staging and cytoreduction. The concept of staging surgery in early stages has its justification in the fact that up to $11 \%$ of "early ovarian cancers" will have metastasis in different sites of the peritoneal cavity at the time of diagnosis. In advanced stages of epithelial ovarian cancer, the goal is the complete cytoreduction of all visible macroscopic disease, since this variable is the most strongly associated with increased overall survival and disease-free period. The ideal time for cytoreductive surgery in relation to chemotherapy (before or after) is still under debate. In 2010 a randomized trial (EORTC) was published, comparing 310 patients initially operated (followed by adjuvant chemotherapy) versus 322 patients initially treated with neoadjuvant chemotherapy (followed by cytoreductive surgery); no significant differences in overall survival between groups were found. Another important factor playing a role in survival and in the probability of surgical cytoreductive success is tumor biology; there has been described a clear difference between serous and mucinous tumors, but some groups advocate that maximal surgical effort in mucinous tumors may compensate morbidity with an increase in survival. The extension of resection in cytoreduction is still controversial; some authors have confirmed that the most important factor is the residual disease and that radical surgery is superior to non-radical surgery in terms of overall survival. The need and extent of lymphadenectomy in advanced cancer will be treated in another chapter of this issue. Undoubtedly, an important factor is to perform procedures in specialized centers.
\end{abstract}

Keywords: Cytoreduction; ovarian epithelial cancer; radical surgery; staging

Submitted Jan 21, 2020. Accepted for publication Aug 25, 2020.

doi: $10.21037 /$ cco-20-40

View this article at: http://dx.doi.org/10.21037/cco-20-40

\section{Introduction}

Ovarian cancer is the sixth most common cancer in women. In developed countries it is considered as the leading cause of death secondary to gynecologic malignancies, with a toll of approximately 22,000 new cases and 15,000 deaths per year $(1,2)$. The worldwide incidence estimate is put at approximately 200,000 new cases with an annual mortality rate of $100,000(3)$.
Most of the ovarian tumors are of epithelial origin (2), where $70-75 \%$ are diagnosed in advanced stage and $25-30 \%$ in early stage (4). According to FIGO, staging is the most important prognostic indicator. The staging is usually carried out by surgical procedure; nevertheless, the performance of imaging studies is crucial prior to such intervention, as this will help in planning the studies and in determining the accurate type of procedure (5). 
The treatment of ovarian cancer should be appropriate, since clinical and surgical decisions may affect the prognosis. Sticking to standard treatment recommendations, such as the performance of the surgery by an expert surgeon (surgical or gynecological oncologist), would guarantee a superior survival result than if the surgery is carried out by inexpert surgeon. The primary objective of ovarian cancer treatment hinges on controlling the disease and on alleviating the symptoms to the greatest extent possible. The extension of the surgery is defined by clinical stage of the disease, the histology, the biology and the clinical characteristics of the patient (6).

Ovarian cancer surgery has witnessed a progressive advance over the years. It plays a fundamental role and its main functions are cancer staging and cytoreduction. The cytoreductive surgery could take place in different moments of the treatment and consists of primary cytoreductive surgery, interval cytoreduction, second-look surgery and secondary cytoreductive surgery. In all these surgeries, the crucial point is to eliminate all the macroscopic diseases, that is, leaving no residual diseases, a condition that is known as R0 (7).

\section{Principles of surgical management in ovarian cancer}

\section{Staging and cytoreduction}

Surgery for the treatment of ovarian cancer can be classified into simple surgery and radical surgery, both of which are part of primary cytoreductive surgery (3). The concept of staging surgery in early stages has been developed as the understanding of the disease and its natural history increased. It was observed that the possibility of metastasis in different sites of the peritoneal cavity could be up to $11 \%$; the possibility of metastasis in the omentum was reported to be $35 \%$, while in peritoneal lavage, the possibility of malignant cells was $33 \%$. Other possible sites of metastasis are the lymph nodes, where the possibility of the occurrence of this, specifically in aortic node, was observed to be $2-24 \%$ in early stages of the disease and in up to $8-15 \%$ of the cases there were metastases in pelvic iliac nodes (4).

In the vast majority of the patients, simple surgery; consisting of hysterectomy and bilateral salpingooophorectomy, infracolic omentectomy, limited excision of retroperitoneal node and segmental resection of small intestine; is performed with minimal possibility of complications.

The radical surgery involves more extensive procedures that are associated with greater risk of bleeding, longer surgery time, longer hospital stay, as well as greater risk of complications which may be decreased with adequate intra and postoperative care (3). Ca125 level has been the most used marker for the diagnosis of epithelial ovarian cancer, and for the detection of recurrences of the disease. However, this marker has a limited utility, since in patients with poor histological prognosis and in early stages, it has not demonstrated a good predictive value (8).

Tumor markers, imaging and laparoscopic studies have been suggested to optimally predict the possibility of tumor resection. In some studies, the elevation of the tumor markers Ca125 and HEA4 has been used as predictors of possible optimal or sub-optimal cytoreduction. Other studies have proposed tomographic findings for the prediction of the impossibility of optimal cytoreduction. Among such findings are massive ascites, hepatic parenchyma metastases, extensive diaphragmatic disease and some others. Laparoscopy allows direct observation of the disease extension. In any case, the decision to perform surgery or give neoadjuvant chemotherapy should be taken by a multidisciplinary team that includes surgical and clinical oncologists (9).

\section{Surgical treatment in initial stages}

The objective of surgery in epithelial ovarian cancer, as depicted before, is to achieve total elimination of all macroscopic disease; the primary treatment in patients with diagnosis of ovarian cancer is surgical (6).

In initial stages, the surgery must be staging (stratification) and should be performed under the following recommendations: peritoneal lavage; inspection and palpation of all the peritoneal surface; bilateral salpingooophorectomy and total hysterectomy; biopsy of any suspicious area; biopsy or resection of any adhesion adjacent to the tumor; infracolic omentectomy; random biopsies of the uterine fundus, bladder peritoneum, right and left pelvic walls, ovarian fossa, right and left colic canals, and hemidiaphragms; and pelvic lymphadenectomy, para-aortic and paracaval lymph nodes (4).

The surgery will provide adequate information for staging (stratification) as well as prognostic information, and will define if adjuvant chemotherapy is needed. An incidental diagnosis may be obtained and if this occurs, postoperative evaluation is required. Usually in this staging 
surgery scenario, less than $30 \%$ of the patients with macroscopic disease are stratified with advance stage of the disease after the surgery. The staging surgery will change its extension and radicalism according to the characteristics of the tumor and of the patient; in mucinous tumors, appendectomy should be performed; fertility-sparing surgery should be considered in patients who still have the desire for fertility, and only under the following conditions: their disease is found in stages $1 \mathrm{~A}$ to $1 \mathrm{C}$ and only one ovary is affected; have favorable histological characteristics such as mucinous and endometrioid grade 1 and 2; and the uterus and an ovary is preserved; in this cases, the rest of the staging surgery is completed (5).

Lymphadenectomy is recommended in patients with serous epithelial tumors, but not in patients with mucinous tumors. Schmeler et al. [2010] published an experience in 107 patients, of which no one received chemotherapy prior to surgical treatment; $87 \%$ of the patients had disease confined to the ovary and the remaining had extra ovarian disease. They carried out lymphadenectomy in $55 \%$ of the patients with confined ovarian disease and in $43 \%$ of the patients with extra ovarian disease; in both groups, the lymph node metastases were negative (10). Mueller published a cohort of 222 patients in which $43 \%$ were subjected to lymphadenectomy; among these patients, only $5 \%$ and $7 \%$ had disease affectation of pelvic and paraaortic nodes respectively without impact on the overall survival (11). In another retrospective analysis carried out by Sebastien Gouy in 114 patients, it was found that only 4 patients had metastases in the lymph nodes. Hence, it was concluded that the performance of lymphadenectomy provides a prognostic value, but not a therapeutic value (12). Based on this, it was concluded not to carry out lymphadenectomy systemically in these patients.

\section{Surgical treatment in advanced stages}

In advanced stages of epithelial ovarian cancer, the goal is complete cytoreduction of all visible macroscopic disease, since this is what has been associated with increased overall survival and disease-free period. In order to achieve this objective, maximum effort has to be made, including intestinal resection, peritonectomy, diaphragmatic resection, retroperitoneal nodes resection and splenectomy, all without increasing the morbidity (13).

The surgical result in epithelial ovarian cancer is usually classified according to residual disease. Complete resection means leaving no residual disease; if there is residual disease, it is classified according to the size of such disease; optimal cytoreduction is when a maximum residual disease diameter of up to $1 \mathrm{~cm}$ is achieved, while sub-optimal cytoreduction is when a greater diametric size of residual disease is achieved (14).

Chemotherapy for ovarian cancer treatment has significantly evolved after the introduction of taxanes in first lines of treatment for ovarian cancer; after 1996 a lot of studies (mainly transversal non randomized) have been published and have proposed that performing debulking surgery after chemotherapy, in advanced stages, could have advantages in the number of optimal cytoreductions achieved. Three randomized clinical trials took place between 2010 and 2019 and their results are interesting in terms of percentages of optimal cytoreductions achieved but failed to demonstrate a significant difference in overall survival and in disease free survival in patients treated with chemotherapy before or after surgery. So, the ideal time for cytoreductive surgery in relation to chemotherapy is still under debate (5). Vergote published in 2010 the results of a randomized study of patients with diagnosis of primary epithelial ovarian cancer with extension to peritoneum or tubes in clinical stages IIIC and IV, analyzing the role of surgery and the surgery time in 632 patients. Initial stage surgery was performed in 310 patients followed by adjuvant chemotherapy, 322 patients initially received neoadjuvant chemotherapy followed by interval surgery; follow-up at 29-30 months concluded that results were similar in both groups. The overall survival according to residual disease, without disease, disease less than $10 \mathrm{~mm}$ in diameter and greater than $10 \mathrm{~mm}$ in diameter were 38,27 , and 25 months for those with residual disease treated with initial chemotherapy and 45, 32 and 26 months for those with the same condition subjected to initial surgery (1).

A meta-analysis included 3 prospective randomized phase 3 studies in advanced epithelial ovarian cancer: AGOOVAR, GINECO and a multicenter study of the French group, with similar inclusion criteria; in the three studies the primary objective was to analyze the impact of surgery in the treatment of advanced epithelial ovarian cancer. A total of 3,388 patients were recruited for the study, out of which a total of 3,126 were included for the analysis. The mean disease-free period was 18.2 months (95\% Cl: 17.3-19.1) and the overall survival was 44.1 months (95\%, CI: $42.3-$ 46.4). At 5 years, disease-free period was $22.6 \%$ and the overall survival was $39 \%$. In this analysis, positive results are observed in the different groups and the conclusion is that surgery plays a fundamental role when complete resection 
of the disease is achieved in all the subgroups. Median survival in group A without any visible residual tumor was 99.1 months (95\% CI, 83.5 to -), the corresponding median survival durations for group B with residual tumor 1-10 mm and group $\mathrm{C}$ with residuals $>10 \mathrm{~mm}$ were 36.2 (95\% CI, 34.6, 39.4) and 29.6 (95\% CI, 27.4, 32.2) months, respectively (log rank test: $\mathrm{P}<0.0001)$. The hazard reduction associated with complete resection versus any residual tumor were very similar for PFS and OS and were $66 \%$ for PFS compared with $68 \%$ for OS. Another important factor found in the analysis was the tumor biology which plays an important role, as is the case of mucinous tumor, which is an important independent factor for the cancer treatment outcome (14).

Seemingly, tumor biology does not only determine survival, but also the surgical outcome. A number of studies have shown that adequate cytoreductive surgery can compensate the results in situations of unfavorable tumor biology.

Some doctors do not advocate for the performance of radical surgery, on the argument that it is fraught with high rate of morbidity. While it is true that the morbidity is high $(25-30 \%)$, the survival rate in a successful surgery makes the morbidity tolerable (3).

Among all the surgical procedures performed in a cytoreductive surgery, only the extensive peritoneum surgery is predictive of survival. The resection of diaphragmatic peritoneum and the entire thickness of the diaphragm have not demonstrated to have an adverse effect in survival results. In a published article on the experience of Mayo Clinic, an important increase in survival was observed in patients who have undergone resection of the diaphragm (53\%) compared to those who did not $(15 \%)$. The same team of researchers reported that the patients operated by surgeons that tend to be more radical had a better survival results than those subjected to non-radical procedures (44\% vs. 17\%).

The extension of resection in cytoreduction is still controversial. Aletti published in 2006 a retrospective work carried out in more than 194 patients with a follow-up of 64.4 months; the objective of the study was to analyze the effect of radical surgery on survival. He observed that the most important factor is the residual disease and that radical surgery is superior to non-radical surgery in terms of overall survival $(3,15)$.

One of the topics that concerns surgeons is the possibility of cytoreduction and the morbidity which the performance of radical surgery entails; in some countries, only few gynecologists are disposed to perform extensive surgery of the upper abdomen. For instance, in China, a publication in 2017 retrospectively described cytoreductive surgery of the upper abdomen carried out from 2009 to 2015 in a total of 150 patients with clinical stage IIIC of epithelial ovarian cancer, majority of which was the serous type. Patients that received chemotherapy prior to the surgery were 14 . One of the conclusions of the study was that an adequate knowledge of the upper abdominal region is fundamental for a successful surgery. They analyzed two groups; in the first one, a complete resection of the diaphragmatic thickness was performed and in the other only peritonectomy was done. The results in both groups were found to be similar; the most common complication was pleural effusion and the application of pleural tube was in $5.3 \%$ compared with $14.6 \%$ in postoperative period; they concluded that the resections of peritoneum or complete diaphragmatic thickness has an acceptable complication rate (16).

Complications in the postoperative period entitles a greater risk of death; apart from this, the cost of hospitalization, readmission, and the time it takes for the patient to return to work should be taken into account; hence, it is necessary to consider the patient's quality of life. In October, 2019, Mayo Clinic published a retrospective review of complications taking place throughout the subsequent 30 days of postoperative period in a cohort of 1,434 patients. In this report, it was observed that the greatest risk factor for a prolonged hospital stay was the leakage of anastomosis with population attributable risk of $33.4 \%$ (95\% CI: $22.3-45.6 \%$ ); therefore, surgeons must focus on reducing the occurrence of anastomosis leakage, as a priority (16).

Undoubtedly, an important factor is to perform procedures in specialized centers $(17,18)$. In a meta-analysis, Cochrane analyzed the morbidity of cytoreductive surgery, as well as the risk of complications in patients that received chemotherapy prior to surgical intervention and did not find difference either in the overall survival or in the progressionfree period. However, regarding the post-treatment quality of life, there was very little evidence, since the reports were limited to adverse effects. Also, they found with limited evidence a reduction in the need for transfusion, stoma and intestinal resection and the likelihood of death was reduced in post-operative period (16).

Another meta-analysis including 17 studies that involved 1,328 patients, published in 2019 by the team of Dublin University, reported the morbidity and mortality associated with initial cytoreductive surgery in comparison with initial neoadjuvant chemotherapy, without finding any significant difference either in surgical time or in infection 
rate. However, they found significant differences in regard to optimal cytoreduction, favoring patients treated with chemotherapy followed by interval surgery; additionally, they found a higher risk of complications $(21.2 \%)$ in patients initially operated versus $8.8 \%$ in the patients who received preoperative chemotherapy before undergoing initial cytoreduction; all the above without significant difference in the overall survival (19).

Regarding lymphadenectomy in advanced epithelial ovarian cancer, the discussion will be treated in another chapter of this issue; however, we should not lose sight of the fact that, in the same way, it should be considered in cytoreductive surgery.

\section{Key points}

The primary treatment for epithelial ovarian cancer consists in adequate staging and well performed surgery.

The surgeon should include in the surgery report: (I) extension of the initial disease; (II) residual disease; and (III) if complete or incomplete resection was performed, with adequate description of the lesions (20).

Surgery should be performed with the intention of not leaving residual disease or to leave disease lesser than $1 \mathrm{~cm}$; this is known as optimal cytoreduction; however, the main objective must be not to leave any visible macroscopic disease.

Cytoreductive surgery with the objective of complete disease resection can be performed by carrying out radical pelvic resection, intestinal resection, appendectomy, lymphadenectomy, deep diaphragmatic or surface diaphragmatic resection, splenectomy, partial hepatectomy, partial gastrectomy, cystectomy or ureteroneocystostomy, cholecystectomy and distal pancreatectomy. The indicated standard route is laparotomy. In patients who have adrenal disease, porta hepatis disease, internal breast chain or supraclavicular disease; the resection of these sites should be performed (21).

One of the points to be emphasized is that in this type of surgery, the procedures should be performed by expert surgeons and with the specialized multidisciplinary team; it has been observed that the patients treated in hospitals where there are high volume of cytoreductive surgeries have lesser risk of complications. In a retrospective analysis published by the team of Johns Hopkins Hospital, an increase in the number of ovarian radical surgery with less number of complications was reported in centers with high volume $(21.7 \%$ vs. $10.2 \%, \mathrm{P}=0.01)(22,23)$.
Therefore, to perform staging (stratification) surgery in epithelial ovarian cancer, several factors such as the patient's age, the desire for fertility, the histologic type and the clinical stage should be taken into account; to perform a cytoreductive surgery, the functional state of the patient and the center where the patient is hospitalized should be considered, since these factors significantly impact in the reduction of postoperative complications and mortality, without affecting the overall survival and the disease progression-free period.

\section{Acknowledgments}

Funding: None.

\section{Footnote}

Provenance and Peer Review: This article was commissioned by the Guest Editor (Heriberto Medina-Franco) for the series "Ovarian Cancer" published in Chinese Clinical Oncology. The article was sent for external peer review organized by the Guest Editor and the editorial office.

Conflicts of Interest: Both authors have completed the ICMJE uniform disclosure form (available at http://dx.doi. org/10.21037/cco-20-40). The series "Ovarian Cancer" was commissioned by the editorial office without any funding or sponsorship. The authors have no other conflicts of interest to declare.

Ethical Statement: The authors are accountable for all aspects of the work in ensuring that questions related to the accuracy or integrity of any part of the work are appropriately investigated and resolved.

Open Access Statement: This is an Open Access article distributed in accordance with the Creative Commons Attribution-NonCommercial-NoDerivs 4.0 International License (CC BY-NC-ND 4.0), which permits the noncommercial replication and distribution of the article with the strict proviso that no changes or edits are made and the original work is properly cited (including links to both the formal publication through the relevant DOI and the license). See: https://creativecommons.org/licenses/by-nc-nd/4.0/.

\section{References}

1. Vergote I, Trope CG, Amant F, et al. Neoadjuvant chemotherapy or primary. surgery in stage IIIC or IV 
ovarian cancer. N Engl J Med 2010;363:943-53.

2. Vitale SG, Marilli I, Lodato M, et al. The role of cytoreductive surgery in advanced-stage ovarian cancer: a systematic review. Updates Surg 2013;65:265-70.

3. Bristow RE. Primary cytoreductive surgery for advanced stage ovarian cancer: indications for radical resection. Cancerol 2007;2 suppl 1:s31-6.

4. Trimbos JB. Surgical treatment of early-stage ovarian cancer. Best Pract Res Clin Obstet Gynaecol 2017;41:60-70.

5. Ledermann JA, Raja FA, Fotopoulou C, et al. Newly diagnosed and relapsed epithelial ovarian carcinoma; ESMO Clinical Practice Guidelines for diagnosis, treatment and follow-up. Ann Oncol 2013;24 suppl 6:vi24-32.

6. Matulonis UA, Sood AK, Fallowfield L, et al. Ovarian Cancer. Nat Rev Dis Primers 2016;2;16061.

7. Lheureux S, Braunstein M, Oza AM. Epithelial ovarian cancer: evolution of management in the era of precision medicine. CA Cancer J Clin 2019;69:280-304.

8. Moon JH, Lee HJ, Kang WD, et al. Prognostic value of serum CA-125 in patients with advanced epithelial ovarian cancer followed by complete remission after adjuvant chemotherapy. Obstet Gynecol Sci 2013;56:29-35.

9. Arab M, Jamdar F, Sadat Hosseini M, et al. Model for prediction of optimal debulking of epithelial ovarian cancer. Asian Pac J Cancer Prev 2018;19:1319-24.

10. Schmeler KM, Tao X, Frumovitz M, et al. Prevalence of lymph node metastasis in primary mucinous carcinoma of the ovary. Obstet Gynecol 2010;116:269-73.

11. Mueller JJ, Lajer H, Mosgaard BJ, et al. International study of primary mucinous ovarian carcinomas managed at tertiary medical centers. Int J Gynecol Cancer 2018;28:915-24.

12. Gouy S, Saidani M, Maulard A, et al. Staging surgery in early-stage ovarian mucinous tumors according to expansile and infiltrative types. Gynecol Oncol Rep 2017;22:21-5.

13. Aletti GD, Dowdy SC, Gostout BS, et al. Quality improvement in the surgical approach to advanced ovarian cancer: the Mayo Clinic experience. J Am Coll Surg 2009;208:614-20.

14. du Bois A, Reuss A, Pujade-Lauraine E, et al. Role of surgical outcome as prognostic factor in advanced epithelial ovarian cancer: a combined exploratory analysis of 3 prospectively randomized phase 3 multicenter trials: by the Arbeitsgemeinschaft Gynaekologische Onkologie Studiengruppe Ovarialkarzinom (AGO-OVAR) and the Groupe d'Investigateurs Nationaux Pour les Etudes des
Cancers de l'Ovaire (GINECO). Cancer 2009;115:1234-44.

15. Aletti GD, Dowdy SC, Gostout BS, et al. Aggressive surgical effort and improved survival in advanced-stage ovarian cancer. Obstet Gynecol 2006;107:77-85.

16. Ye S, He T, Liang S, et al. Diaphragmatic surgery and related complications in primary cytoreduction for advanced ovarian, tubal, and peritoneal carcinoma. BMC Cancer 2017;17:317.

17. Narasimhulu DM, Bews KA, Hanson KT, et al. Using evidence to direct quality improvement efforts: defining the highest impact complications after complex cytoreductive surgery for ovarian cancer. Gynecol Oncol 2020;156:278-83.

18. Wimberger P, Lehmann N, Kimmig R, et al. Prognostic factors for complete debulking in advanced ovarian cancer and its impact on survival: an exploratory analysis of a prospectively randomized phase III study of the Arbeitsgemeinschaft Gynaekologische Onkologie Ovarian Cancer Study Group (AGO-OVAR). Gynecol Oncol 2007;106:69-74.

19. Morrison J, Haldar K, Kehoe S, Lawrie TA. Chemotherapy versus surgery for initial treatment in advanced ovarian epithelial cancer. Cochrane Database Syst Rev 2012;2012:CD005343.

20. Bartels HC, Rogers AC, McSharry V, et al. A meta-analysis of morbidity and mortality in primary cytoreductive surgery compared to neoadjuvant chemotherapy in advanced ovarian malignancy. Gynecol Oncol 2019;154:622-30.

21. Cliby WA, Powell MA, Al-Hammadi N, et al. Ovarian cancer in the United States: contemporary patterns of care associated with improved survival. Gynecol Oncol 2015;136:11-7.

22. Jeon S, Lee SJ, Lim MC, et al. Surgical manual of the Korean Gynecologic Oncology Group: ovarian, tubal, and peritoneal cancers. J Gynecol Oncol 2017;28:e6.

23. Sinno AK, Li X, Thompson RE, et al. Trends and factors associated with radical cytoreductive surgery in the United States: a case for centralized care. Gynecol Oncol 2017;145:493-9.

Cite this article as: Hernandez-Lopez LA, Elizalde-Mendez A. How far should we go in optimal cytoreductive surgery for ovarian cancer? Chin Clin Oncol 2020;9(5):70. doi: 10.21037/ cсо-20-40 